\title{
Investigaciones en el desierto de Atacama Centro UC Desierto de Atacama - Estación Atacama UC, Oasis de Niebla Alto Patache
}

\author{
Juan Luis García y Pablo Osses²
}

El desierto de Atacama posee un conjunto de elementos que lo hacen significativo a nivel mundial, por nombrar algunos podemos mencionar que efectivamente es el más seco del mundo, con menos de $1 \mathrm{~mm}$ de precipitaciones promedio anuales, tal vez por esta misma razón es el sitio del planeta que mejor permite la exploración del universo, contando con un impresionante conjunto de observatorios astronómicos de relevancia mundial, posee las más alta y extendida porción montañosa de los Andes solo asimilable a los Himalaya y el Karakoram.

En un contexto país, para Chile ha sido la principal fuente de riqueza económica sin lugar a dudas, a través de la explotación de sus recursos naturales, especialmente minería. Tenemos el periodo de las guaneras, luego el salitre para finalmente desarrollar el cobre que conocemos actualmente, sumado a esto no es extraño que miremos hacia el futuro pensando en la minería no metálica, con casos como el litio, más aún si incorporamos el enorme desarrollo de las tecnologías eléctricas, las fuentes solares y la necesidad de almacenamiento. La suma de lo anterior nos da más de 200 años en que este territorio ha generado cerca de la mitad de la producción agregada nacional.

Esta inmensa relevancia geográfico económica y por lo tanto social, no ha pasado desapercibida en la historia chilena, tal es así que durante el siglo XIX el Estado de Chile contrató a un conjunto importante de especialistas europeos para poder generar una base de conocimiento que permitiera hacerse una idea de que es lo que realmente hay en este enorme territorio llamado Atacama, en esa lista podemos mencionar a Claudio Gay, Amado Pissis, Ignacio Domeyko, Rodulfo Phillipi entre otros sabios que recorrieron a pie y en lomo de mula las vastedades del desierto para catastrar cada una de sus partes, en términos cartográficos, geológicos, vegetacionales, sociales y económicos.

En la segunda mitad del siglo XX, es la Corporación de Fomento de la Producción (CORFO) que como su nombre bien lo señala, tiene por misión fomentar la industrialización y desarrollo del país. En este contexto es que nuevamente mira hacia el desierto como un territorio lleno de oportunidades para sus objetivos, apoyando en esos años la minería, el desarrollo de fundiciones y prospecciones, para actualmente, ya en pleno siglo XXI ser un pilar fundamental en el desarrollo energético, especialmente solar.

Centro UC Desierto de Atacama / Instituto de Geografía, Pontificia Universidad Católica de Chile (Chile). E-mail: jgarciab@uc.cl

Centro UC Desierto de Atacama / Instituto de Geografía, Pontificia Universidad Católica de Chile (Chile). E-mail: posses@uc.cl 
Actualmente, es difícil concebir el desarrollo de los países y sus ciudadanos sin una clara base de conocimiento científico respecto a los componentes y dinámicas geográficas de los territorios, en este sentido es que existen en el mundo un número importante de centros de investigación y estaciones científicas especializadas en ámbitos variados tales como glaciares, montañas, océanos, selvas tropicales, ártico y antártico y obviamente los desiertos no están excluidos de este interés e innegable necesidad. Desde el año 2006 se crea el Centro UC Desierto de Atacama (CDA) de la Pontificia Universidad Católica de Chile para desarrollar investigación científica en temáticas desérticas de alto interés e impacto. El conocimiento fundamental así producido busca ser transmitido a la comunidad local y agentes que toman decisiones territoriales para velar por el desarrollo sustentable del desierto del norte de Chile. Su directora original, precursora y fuente inagotable de inspiración, fue Pilar Cereceda T., la que fue apoyada muy cerca por el distinguido arqueólogo y conocedor del vasto desierto atacameño, Dr. Horacio Larraín B. El CDA hoy sigue siendo integrado por un grupo interdisciplinario de investigadores del campo de la Geografía, Ingeniería, Agronomía, Arqueología, Biología y Arquitectura. Estos investigadores lideran proyectos relacionados con la climatología de nieblas, recursos hídricos, desertificación, energía solar, habitación sustentable y evolución del paisaje desértico y su gente. Una de las líneas de investigación de importancia dentro del quehacer del CDA es contribuir al entendimiento del geo-ecosistema costero del desierto a través de estudios atmosféricos, oceanográficos, ecológicos, arqueológicos, geomorfológicos, presente y pasado. Nuestro laboratorio natural para alcanzar este objetivo esta centrado en la Estación Atacama UC, Oasis de Niebla Alto Patache.

El sector de Alto Patache y su valor cultural y ecosistémico ha formado parte del interés de la UC y sus investigadores desde 1997. Tanto es así que desde 1998 se registra de manera continua la captación de agua de niebla en el lugar, siendo una de las series de tiempo estandarizadas en agua de niebla más largas del país y del mundo. De la relevancia de la niebla como recurso hídrico se han ido desprendiendo y desarrollando un sinnúmero de quehaceres científicos que abordan la arqueología, la botánica, la ingeniería, agricultura, arquitectura e incluso el arte in-situ. Esto ha llevado a un importante crecimiento en las capacidades de infraestructura de la Estación Atacama UC, la que actualmente es laboratorio de campo, plataforma de operaciones que permite proyectarse sobre el desierto costero, sala de clases para estudiantes universitarios de chile y el mundo, así como también para un conjunto importante de escolares de la región de Tarapacá.

El foco de este número especial de la Revista de Geografía Norte Grande es la investigación en la Estación Atacama UC y es reflejo, al menos parcialmente, de la actividad del CDA y sus colaboradores en el desierto. A través de los cinco trabajos que se incluyen dentro de esta edición especial, se busca promover la investigación interdiciplinar en el desierto costero y extender la invitación a colaborar en el futuro. La línea común de los tres primeros trabajos es la niebla, también conocida como Camanchaca. Los últimos dos trabajos se enfocan en los efectos de las Iluvias torrenciales del 9 de agosto 2015, año El Niño fuerte, en Alto Patache.

El trabajo Manuel Escobar M. y Magdalena García B., busca profundizar en el debate sobre el origen de la denominación Camanchaca. En una muy interesante revisión de casos locales y extranjeros, los autores dan a conocer la complejidad e incertidumbre en develar la "arqueología" de los nombres, cómo estos se originan y transforman en el tiempo, cambiando su connotación, y en casos desapareciendo. En el caso particular de Camanchaca se discute el vínculo que puede existir del término tanto como denominador de la abundante y persistente neblina que empapa los cerros del desierto costero versus los antiguos habitantes del lugar, hoy denominados gené- 
ricamente como changos. Así se ilustran las posibilidades de Camanchaca como etnónimo y su etimología. La investigación nos lleva a las primeras menciones en escritos de la colonia (S. XVI) que hacen referencia a los Camanchacos o pescadores del litoral desértico que se distinguían de sus vecinos, por ejemplo, como grupo lingüístico. Después de siglos sin que se haga mención a la denominación a Camanchaca, el término reaparece nuevamente solo en la transición S. XIX-XX para hacer alusión a la nube baja y garúa, principal fuente de humedad atmosférica de la costa del desierto. En una nueva propuesta, los autores plantean que el término Camanchaca nace como un etnónimo que derivaría de ccamachatha, aludiendo a las poblaciones costeras que secaban pescado y, como es sabido, se intercambiaba con las poblaciones del interior. El trabajo concluye con la noción inseparable de Camanchaca la nube y el habitante original, perpetuado en los oasis de niebla costeros, fuentes de recursos terrestres y enclaves de intercambio social, económico y territorial.

El artículo de Pablo Osses, Rodrigo Escobar, Camilo del Río, Redlich García y Constanza Vargas, toma largas series de tiempo en agua de niebla y su caracterización anual, mensual y en algunos casos horaria, junto con los datos de irradiancia levantados por investigadores para el sector superior del acantilado costero en la Estación Atacama UC. Los autores plantean que dado los comportamientos espacio temporales de estos dos recursos (agua y energía), existen una complementariedad y no una anulación, especialmente de la energía solar producto de la atenuación por la presencia de nubosidad. Tal es así que los ciclos diarios de nieblas, tanto orográficas como advectivas, presentan un incremento de presencia y por ende de producción de agua en la madrugada, atardecer y durante la noche, mientras que obviamente la irradiancia tiene una curva de incremento asociada directamente al ángulo solar y la luz diurna, con pequeñas variaciones estacionales. En síntesis los autores concluyen una correlación inversa entre ambos recursos, dando pie a la investigación y potencial implementación de sistemas de producción y manejo de recursos hídricos basados en el uso de energía solar, ampliando con esto el territorio disponible para ser utilizado de manera sustentable en beneficio de los ciudadanos y sus actividades.

El trabajo de Reinaldo Vargas Castillo, Daniel Stanton y Peter R. Nelson, busca llenar el vacío que existe sobre el conocimiento de la biodiversidad de líquenes en la costa del norte de Chile y en general de nuestro país. Los líquenes forman parte de una costra biológica en el oasis de niebla de Alto Patache que tiene la capacidad de estabilizar la capa superficial del suelo y aumentar su capacidad de absorber agua favoreciendo eventualmente al establecimiento de plantas vasculares. La presencia extensiva de estos organismos diminutos, adaptados a condiciones de estrés máxima, determina el enriquecimiento del suelo. El alto grado de especialización y endemismos de los líquenes en Atacama genera que el trabajo de Vargas y equipo en Alto Patache responda a una necesidad urgente de conocer en profundidad la biodiversidad y su historia en los oasis de niebla del Atacama. Alto Patache se presenta entonces como uno de los hot-spots de biodiversidad del Atacama costero gracias al aporte de la camanchaca presente todo el año. Los autores ven en este lugar un gran potencial para el estudio de los líquenes dado que hasta ahora solo se habían desarrollado aproximaciones a las plantas vasculares del sitio, sin considerar en profundidad a los líquenes. Bajo la premisa de que la distribución de la neblina tiene una directa relación con la presencia y expresión espacial de los líquenes, este trabajo presenta una estrategia metodológica ad-hoc que le permite revelar resultados de alto impacto, que, hasta la fecha, incluyen 77 especies de líquenes para Alto Patache, 61 de las cuales corresponden a nuevos registros para la región de Tarapacá y cuatro registros de especies nuevos para el país. Los autores destacan 
la presencia de Santessonia cervicornis en Alto Patache y el rol de la Estación Atacama UC en la conservación de esta especie en "Peligro Crítico de Extinción".

Los dos últimos trabajos asociados a la temática de este numero especial corresponden a aquellos de Héctor Orellana, Juan-Luis García, Carla Ramírez y Nicolás Zanetta, y de Patricio Pliscoff, Nicolás Zanetta, Josefina Hepp y Javiera Machuca, vinculando el efecto de las Iluvias anómalas ocurridas el 9 de agosto del año El Niño 2015 sobre Alto Patache. Mientras el primer trabajo describe en detalle la meteorología del evento pluviométrico y la respuesta hidro-geomorfológica, el segundo trabajo evalúa el efecto de las lluvias en la diversidad de la floración en Alto Patache, caracterizando además la vegetación y su relación con la presencia de la niebla en el área. El trabajo de Orellana y equipo, es un ejercicio por entender la dinámica del desierto de Atacama en cuanto su aparente carácter de estabilidad morfogenética. Los autores aprovechan el registro único de datos meteorológicos en la Estación Atacama UC en Alto Patache de valioso detalle para caracterizar el evento atmosférico de la mañana del 9 de Agosto (9A). Las Iluvias torrenciales que sumaron $51.6 \mathrm{~mm}$ en pocas horas activaron los talwegs secos y produjeron múltiples aluviones en el área. Con una aproximación metodológica múltiple, el trabajo logra describir la anatomía del "9A", diferenciando etapas discretas de desarrollo del fenómeno pluvio-geomorfológico en una sub cuenca de control de Alto Patache y valorando cuantitativamente el arrastre de materiales desde las quebradas. Los autores registran excavaciones de quebradas por bajo los 2 metros de profundidad. Se identifican las facies aluviales propias de estos eventos, en este caso producidas durante el 9A, las que a su vez se contrastan con la estratigrafía expuesta. La evidencia de aluviones antiguos en Alto Patache da cuenta de un fenómeno recurrente en el tiempo, tentativamente ligado a años El Niño y sus lluvias intensas en el desierto de Atacama costero. Quizás aún más importante, se da cuenta que este tipo de tormentas ocurren de forma localizada en el espacio y que de afectar zonas pobladas, como de hecho ocurrió ese día en Tocopilla, esto se traduce en dramáticas pérdidas humanas y materiales. Por ende, se hace un lógico llamado a considerar esta dinámica aluvial súbita e irregular en el tiempo en la planificación de los asentamientos del desierto y así aminorar futuras crisis.

Por su parte, Pliscoff y equipo generan una novedosa aproximación en la descripción de la flora y vegetación de Alto Patache al realizar una descripción en segmentos altitudinales que contrastan con datos de agua de niebla. Ellos identifican un total de 42 especies, 32 corresponden a especies endémicas de Chile. Su estudio incluye una especie del genero Tiquilia (probablemente T. litoralis), especie que se ha descrito para la costa desértica de Chile, pero no colectada en el Oasis de Niebla Alto Patache previamente. Los autores refuerzan la idea de que las lluvias asociadas a El Niño son una oportunidad de reproducción, dispersión y establecimiento para estas especies, así como para renovar el banco de semillas del suelo y así mantener la biodiversidad propia del Oasis de Niebla de Alto Patache. El trabajo concluye que no hay una relación directa entre intensidad de niebla y riqueza florística, siendo más bien la niebla una mantenedora de la vegetación arbustiva en el acantilado costero.

Junto a estos con estos artículos, se publican otros cuatro trabajos que abordan diversas temáticas y problemas geográficos. El primero de ellos, de Pablo Camus, Rodrigo Hidalgo, Leonel Pérez y Enrique Muñoz, plantea que, en la historiografía y geografía histórica chilena, no se ha reflexionado aún sobre los procesos y conflictos territoriales que han operado en los espacios litorales y marítimos. Estudiando un conflicto suscitado antes de promediar el siglo XX en la lo- 
calidad costanera de Dichato, actual región del Biobío, los autores concluyen que este problema entre propietarios, pescadores, habitantes costaneros, veraneantes y supuestos concesionarios fiscales por el uso de la franja litoral, ejemplifica e ilustra claramente como las disputas territoriales por los bienes comunes pueden llegar a lograr la generación de estrategias y mecanismos de adaptación que en su momento interrumpan los procesos globales de apropiación, cercamiento y destrucción de los bienes y espacios comunes.

El texto de Raúl Molina Otárola y Luis Campos Muñoz, explora el desarrollo de una etnogénesis diaguita en el valle del río Huasco, región de Atacama, Chile. Los autores señalan que este proceso tiene su base histórica y territorial en los pueblos de indios formados en el periodo colonial, en los siglos XVII y XVIII. Esto permite manifestar que se trata de la autoafirmación de una identidad individual y colectiva, que reconoce un pasado indígena y una memoria espacial, que sustentan este proceso de reconocimiento íntimamente vinculado al territorio. Ante esto, afirman que en el Huasco Alto se ha recreado una identidad indígena en el marco de un confín geográfico, cuyas características orográficas y ambientales permitieron constituirlo en lugar de refugio, zona de reproducción cultural y productiva, que ayudó a sus habitantes a conservar las tierras y mantener el poblamiento ancestral.

Héctor Mendoza Vargas explora el surgimiento en México de la revista MAPA entre 1934 y 1940. Esta publicación ordenaba y actualizaba la información sobre los lugares y sobre la manera de viajar con base en las fotografías, las instrucciones y los mapas. Las páginas de la revista formaban una especie de álbum geográfico con una variedad de opciones señaladas a los particulares, en consonancia con la motorización de la vida privada y el impulso nacionalista del gobierno. El autor señala que las tres secciones elegidas de la revista MAPA (Caminos, Automovilismo, y Viajes y excursiones) eran representativas de la diversidad de México y de las opciones paisajísticas entre los lectores. De esta manera, desde la Ciudad de México se abrió un canal de comunicación a través de la revista MAPA que transformaba la mirada de los viajeros ante la mayor variedad de rasgos naturales y humanos registrados, en este sentido, la edición respaldaba la construcción de los paisajes mexicanos.

Bernd Pfannenstein, Edgar Eduardo Anacleto Herrera y Salvador Sevilla Villalobos, analizan el fenómeno de las urbanizaciones cerradas en el Área Metropolitana de Guadalajara, México. Frente a la masividad de estas tipologías constructivas, los autores se preguntan si ilas urbanizaciones cerradas son el modelo correcto para afrontar la carencia técnica y operacional que la administración pública tiene en el cumplimiento de su deber con la ciudadanía? o si, por el contrario, ¿son solo un producto de consumo exitoso que fue bien recibido por las poblaciones latinoamericanas de altos recursos económicos? Concluyen que la población que está optando por la vida fortificada, pues percibe una mejoría en su estilo de vida ya que se ha resuelto en cierta medida la incapacidad del Estado para cubrir necesidades básicas. Sin embargo, este modelo está generando más dificultades que beneficios, ya que la sociedad se segmenta cada vez más, se incrementa la distancia entre ricos y pobres y surgen nuevos estratos sociales.

El artículo de Danny Alexander Cocuñame Ricardo y Elkin de Jesús Salcedo Hurtado, establecen la estimación del riesgo de inundación por tsunamis local en la isla de Cascajal, localizada en el Pacífico colombiano, a partir de la generación de eventos sísmicos tsunamigénicos de orden probabilístico, los cuales se establecen por medio de un análisis de amenaza sísmica para las 
fuentes de subducción en el área de estudio. Concluyen que, para la isla de Cascajal, el escenario de inundación por tsunami presenta valores de inundación heterogéneos que afectarían de manera significativa la población e infraestructura urbana de dicha zona. Frente a esto, recomiendan realizar una urgente actualización de la información primaria en terreno, debido a que los datos utilizados tienen fecha de expedición del último censo nacional (2005), para poder actualizar los parámetros de vulnerabilidad y riesgo de la isla.

En la sección reseña de este número semitemático se publica una entrevista que realizaron Juan-Luis García y Pablo Osses a los académicos Pilar Cereceda y Horacio Larraín, fundadores e inspiradores del Centro UC Desierto de Atacama, con el objetivo de indagar sobre los primeros acercamientos y estudios que se realizaron en el Oasis de Niebla Alto Patache. Así también, se les consulta sus perspectivas sobre los desafíos, tanto en investigación, educación y difusión, que aún quedan por realizar en este interesante lugar.

Por último, Rosa Inés Babilonia Ballesteros nos reseña el libro "Re-conociendo las geografías de América Latina y el Caribe", editado por Rafael Sánchez, Rodrigo Hidalgo y Federico Arenas durante este año. El texto constituye un homenaje a las contribuciones realizadas por el geógrafo alemán Dr. Axel Borsdorf, recientemente retirado de la vida académica, quien investigó diversas temáticas sobre nuestro continente durante los últimos 40 años. 